\title{
Balanced truncation model reduction of periodic systems
}

\author{
A. Varga \\ German Aerospace Center, DLR - Oberpfaffenhofen \\ Institute of Robotics and Mechatronics \\ D-82234 Wessling, Germany. \\ Andras. Varga@dlr.de
}

\begin{abstract}
The balanced truncation approach to model reduction is considered for linear discrete-time periodic systems with time-varying dimensions. Stability of the reduced model is proved and a guaranteed additive bound is derived for the approximation error. These results represent generalizations of the corresponding ones for standard discrete-time systems. Two numerically reliable methods to compute reduced order models using the balanced truncation approach are considered. The square-root method and the potentially more accurate balancing-free square-root method belong to the family of methods with guaranteed enhanced computational accuracy. The key numerical computation in both methods is the determination of the Cholesky factors of the periodic Gramian matrices by solving nonnegative periodic Lyapunov equations with time-varying dimensions directly for the Cholesky factors of the solutions.
\end{abstract}

\section{Introduction}

In this paper we address the balanced truncation (BT) model reduction approach of linear periodic discretetime systems with time-varying dimensions. There are several reasons to consider a time-varying state dimensions setting for studying the model reduction problems of periodic systems. The first reason is that generally the minimal (i.e., reachable and observable) realization of a periodic system with constant dimensions can have a periodically varying state dimension $[5,4,18]$. For such system the model reduction problem can not be generally solved by using, for example, a pure balancing based approach since a minimal balanced realization with constant dimensions may not exist even if the original system has constant dimensions. Methods using linear matrix inequalities (LMIs) were used in [9] with the same goal of avoiding the need for existence of minimal balanced realizations. On contrary, a minimal balanced realization with time-varying dimensions always exists [18]. A second reason is that the proper formulation of the periodic model reduction problem generally produces reduced order models with time-varying dimensions. For example, imposing standard conditions for the reduced system as stability, minimality and an a priori specified bound for the approximation error, leads usually to a periodically time-varying state dimension for the reduced order periodic system. Trying to fulfill the same error bound by imposing constant dimensions results usually in higher order approximations. Finally, allowing varying dimensions helps to develop completely general numerical methods based on truncation formulas able to address the reduction of nonminimal stable periodic systems as well. Such a feature is not only desirable but is obligatory for a satisfactory numerical method, otherwise even systems which are nearly nonminimal can not be properly handled. Note that all methods based on direct truncation formulas to compute reduced order periodic systems can fail when applied to nonminimal systems unless timevarying dimensions are allowed.

In this paper we describe the BT model reduction approach for periodic systems with time-varying dimensions and generalize well known results on stability and minimality of the reduced system [13], and the guaranteed approximation error bounds for standard discretetime systems [1] to the periodic setting. For complete generality we also allow time-varying dimensions for input and output vectors. Our approach is based on reformulating the periodic model reduction problem as a standard model reduction problem for time-invariant discrete-time systems via the cyclic reformulation technique introduced in [12]. The derived error bound for time-varying dimensions is essentially the same as the bound derived in [9] using an LMIs-based balancing approach for constant dimension systems.

We also discuss numerical approaches to compute reduced order periodic systems by the BT method. A first method belongs to the family of square-root methods with guaranteed enhanced computational accuracy. The matrices of the reduced periodic system are computed using appropriate truncation matrices determined exclusively using the Cholesky factors of the periodic reachability and observability Gramians. This method is a generalization of the square-root method for standard systems [15] to the periodic case. An alternative balancing-free square-root approach has 
the advantage of a potentially better numerical accuracy in case of poorly scaled original systems. By avoiding the use of possibly ill-conditioned balancing based truncation formulas and by using instead wellconditioned truncation matrices, the accuracy of computations can be often significantly improved. This second method extends the balancing-free square-root approach for standard systems [16] to periodic systems. The key computation in both approaches is the solution of nonnegative periodic Lyapunov equations with timevarying dimensions directly for the Cholesky factors of the periodic Gramians. For this purpose, a recently developed numerically reliable computational algorithm is employed [18].

\section{Preliminaries}

Consider the linear discrete-time $K$-periodic system

$$
\begin{aligned}
x_{k+1} & =A_{k} x_{k}+B_{k} u_{k} \\
y_{k} & =C_{k} x_{k}
\end{aligned}
$$

where the matrices $A_{k} \in \mathrm{R}^{n_{k+1} \times n_{k}}, B_{k} \in \mathrm{R}^{n_{k+1} \times m_{k}}$, $C_{k} \in \mathrm{R}^{p_{k} \times n_{k}}$ and the integers $n_{k}, m_{k}, p_{k}$ are periodic with period $K \geq 1$. The transition matrix of the system (1) is defined by the $n_{j} \times n_{i}$ matrix $\Phi_{A}(j, i)=$ $A_{j-1} A_{j-2} \cdots A_{i}$, where $\Phi_{A}(i, i):=I_{n_{i}}$. The state transition matrix over one period $\Phi_{A}(j+K, j) \in \mathrm{R}^{n_{j} \times n_{j}}$ is called the monodromy matrix of system (1) at time $j$ and its eigenvalues are called characteristic multipliers at time $j$. Note that $\Lambda\left(\Phi_{A}(j+K, j)\right)$ has always at least $n_{j}-\underline{n}$ zero elements, where $\underline{n}:=\min _{k}\left\{n_{k}\right\}$. The rest of $\underline{n}$ eigenvalues are independent of time $j$ and form the core characteristic multipliers [6]. The periodic system (1) is asymptotically stable if all characteristic multipliers belong to the open unit disk. For the definitions of reachability, observability and minimality of periodic systems we use the corresponding notions from [5] for general time-varying systems.

Definition 1. The periodic system (1) is reachable at time $k$ if

$$
\operatorname{rank} G_{k}=n_{k}
$$

where $G_{k}$ is the infinite columns matrix

$$
G_{k}=\left[\begin{array}{llll}
B_{k-1} & A_{k-1} B_{k-2} & \cdots & \Phi_{A}(k, i+1) B_{i} \cdots
\end{array}\right] .
$$

The periodic system (1) is completely reachable if (2) holds for all $k$.

Definition 2. The periodic system (1) is observable at time $k$ if

$$
\operatorname{rank} F_{k}=n_{k}
$$

where $F_{k}$ is the infinite rows matrix

$$
F_{k}=\left[\begin{array}{c}
C_{k} \\
C_{k+1} A_{k} \\
\vdots \\
C_{i} \Phi_{A}(i, k) \\
\vdots
\end{array}\right]
$$

The periodic system (1) is completely observable if (4) holds for all $k$.

Definition 3. The periodic system (1) is minimal if it is completely reachable and completely observable.

For an asymptotically stable periodic system, the $n_{k} \times n_{k}$ reachability Gramian at time $k$ is defined as

$$
P_{k}:=\sum_{i=-\infty}^{k-1} \Phi_{A}(k, i+1) B_{i} B_{i}^{T} \Phi_{A}(k, i+1)^{T}=G_{k} G_{k}^{T} \geq 0,
$$

where $G_{k}$ is defined in (3). Similarly, the $n_{k} \times n_{k}$ observability Gramian at time $k$ is defined as

$$
Q_{k}=\sum_{i=k}^{\infty} \Phi_{A}(i, k)^{T} C_{i}^{T} C_{i} \Phi_{A}(i, k)=F_{k}^{T} F_{k} \geq 0 .
$$

with $F_{k}$ defined by (5). Note that both Gramians are $K$-periodic matrices. Using the definitions of reachability and observability we have the following results.

Proposition 1 The periodic system (1) is reachable at time $k$ iff $P_{k}>0$ and is completely reachable iff $P_{k}>0$ for $k=1, \ldots, K-1$.

Proposition 2 The periodic system (1) is observable at time $k$ iff $Q_{k}>0$ and is completely observable iff $Q_{k}>0$ for $k=1, \ldots, K-1$.

Notation. For a $K$-periodic matrix $X_{k}$ we use alternatively the script notation

$$
\mathcal{X}:=\operatorname{diag}\left(X_{0}, X_{1}, \ldots, X_{K-1}\right),
$$

which associates the block-diagonal matrix $\mathcal{X}$ to the cyclic matrix sequence $X_{k}, k=0, \ldots, K-1$. This notation is consistent with the standard matrix operations as for instance addition, multiplication, inversion as well as with several standard matrix decompositions (Cholesky, SVD). We denote with $\sigma \mathcal{X}$ the $K$-cyclic shift

$$
\sigma \mathcal{X}=\operatorname{diag}\left(X_{1}, \ldots, X_{K-1}, X_{0}\right)
$$

of the cyclic sequence $X_{k}, k=0, \ldots, K-1$. By using the script notation, the periodic system (1) will be alternatively denoted by the triple $(\mathcal{A}, \mathcal{B}, \mathcal{C})$ and the timevarying state vector dimensions are denoted compactly by $\mathbf{n}=\left(n_{0}, \ldots, n_{K-1}\right)$. 


\section{Balanced truncation method}

For an asymptotically stable periodic system the two Gramians are nonnegative definite and satisfy nonnegative (or positive) discrete periodic Lyapunov equations (PDPLEs) as follows: the reachability Gramian $\mathcal{P}$ satisfies the forward-time PDPLE

$$
\sigma \mathcal{P}=\mathcal{A} \mathcal{P} \mathcal{A}^{T}+\mathcal{B B}^{T},
$$

while the observability Gramian $\mathcal{Q}$ satisfies the reversetime PDPLE

$$
\mathcal{Q}=\mathcal{A}^{T} \sigma \mathcal{Q A}+\mathcal{C}^{T} \mathcal{C}
$$

Let $T_{k} \in \mathrm{R}^{n_{k} \times n_{k}}$ be a $K$-periodic invertible matrix. Two periodic systems $(\mathcal{A}, \mathcal{B}, \mathcal{C})$ and $(\widetilde{\mathcal{A}}, \widetilde{\mathcal{B}}, \widetilde{\mathcal{C}})$ related by the transformation

$$
(\widetilde{\mathcal{A}}, \widetilde{\mathcal{B}}, \widetilde{\mathcal{C}})=\left(\sigma \mathcal{T}^{-1} \mathcal{A T}, \sigma \mathcal{T}^{-1} \mathcal{B}, \mathcal{C} \mathcal{T}\right)
$$

are called Lyapunov-similar and (8) is called a Lyapunov similarity transformation. The Gramians $\widetilde{\mathcal{P}}$ and $\widetilde{\mathcal{Q}}$ of the transformed system $(\widetilde{\mathcal{A}}, \widetilde{\mathcal{B}}, \widetilde{\mathcal{C}})$ satisfy

$$
\widetilde{\mathcal{P}}=\mathcal{T}^{-1} \mathcal{P} \mathcal{T}^{-T}, \quad \widetilde{\mathcal{Q}}=\mathcal{T}^{T} \mathcal{Q} \mathcal{T} .
$$

For a completely reachable and completely observable (i.e., minimal) periodic system, $\mathcal{T}$ can be determined such that the transformed Gramians are equal and diagonal and thus the transformed periodic system is balanced $[17,18]$. The diagonal elements of the balanced Gramians are called the Hankel singular values and are the positive square-roots of the eigenvalues of the product $\mathcal{P} \mathcal{Q}$. The maximum of them defines the Hankelnorm of the given periodic system [2].

Let $\mathcal{P}=\mathcal{S}^{T} \mathcal{S}$ and $\mathcal{Q}=\mathcal{R}^{T} \mathcal{R}$ be the Cholesky factorizations of Gramians. For a minimal system, in analogy with the standard case [15], we can use the singular value decomposition

$$
\mathcal{R S}^{T}=\mathcal{U} \Sigma \mathcal{V}^{T}
$$

to compute the balancing transformation matrix $\mathcal{T}$ and its inverse $\mathcal{T}^{-1}$ as

$$
\mathcal{T}=\mathcal{S}^{T} \mathcal{V} \Sigma^{-1 / 2}, \quad \mathcal{T}^{-1}=\Sigma^{-1 / 2} \mathcal{U}^{T} \mathcal{R} .
$$

Assume from now that the original periodic system (1) is minimal and balanced and the periodic gramians are partitioned as follows

$$
P_{k}=Q_{k}=\Sigma_{k}=\left[\begin{array}{cc}
\Sigma_{k, 1} & 0 \\
0 & \Sigma_{k, 2}
\end{array}\right],
$$

where $\quad \Sigma_{k, 1}=\operatorname{diag}\left(\sigma_{k, 1}, \ldots, \sigma_{k, r_{k}}\right), \quad \Sigma_{k, 2}=$ $\operatorname{diag}\left(\sigma_{k, r_{k}+1}, \ldots, \sigma_{k, n_{k}}\right)$ and $\sigma_{k, 1} \geq \ldots \geq \sigma_{k, r_{k}}>$ $\sigma_{r_{k}+1} \geq \ldots \geq \sigma_{k, n_{k}} \geq 0$. Let us partition the periodic system matrices accordingly

$$
\begin{gathered}
A_{k}=\left[\begin{array}{ll}
A_{k, 11} & A_{k, 12} \\
A_{k, 21} & A_{k, 22}
\end{array}\right], \quad B_{k}=\left[\begin{array}{l}
B_{k, 1} \\
B_{k, 2}
\end{array}\right], \\
C_{k}=\left[\begin{array}{ll}
C_{k, 1} & C_{k, 2}
\end{array}\right]
\end{gathered}
$$

The reduced order system $\left(\mathcal{A}_{11}, \mathcal{B}_{1}, \mathcal{C}_{1},\right)$ can be considered, analogously to the standard case, as an approximation of the original system of order $\mathbf{r}=\left(r_{0}, \ldots, r_{K-1}\right)$. The following theorem generalizes the results of [13] on stability and minimality of the reduced model.

Theorem 1 Consider the balanced stable periodic system $(\mathcal{A}, \mathcal{B}, \mathcal{C}$,$) of order \mathbf{n}$ and period $K>1$, and let $\left(\mathcal{A}_{r}, \mathcal{B}_{r}, \mathcal{C}_{r},\right):=\left(\mathcal{A}_{11}, \mathcal{B}_{1}, \mathcal{C}_{1},\right)$ be the reduced periodic system of order $\mathbf{r}$ resulted by truncation. Then $\left(\mathcal{A}_{r}, \mathcal{B}_{r}, \mathcal{C}_{r},\right)$ is asymptotically stable and minimal.

Proof. Consider the cyclic shift matrix

$$
Z_{\mathbf{n}}=\left[\begin{array}{cccc}
0 & \cdots & 0 & I_{n_{K-1}} \\
I_{n_{0}} & \cdots & 0 & 0 \\
\vdots & \ddots & \vdots & \vdots \\
0 & \cdots & I_{n_{K-2}} & 0
\end{array}\right]
$$

and define (similar as done in [12] for constant dimensions) the extended (or cyclic lifted) time-invariant system

$$
\left(A_{e}, B_{e}, C_{e}\right):=\left(Z_{\mathbf{n}} \mathcal{A}, Z_{\mathbf{n}} \mathcal{B}, \mathcal{C}\right)
$$

corresponding to the periodic system $(\mathcal{A}, \mathcal{B}, \mathcal{C})$. It can be easily shown that the Gramians corresponding to the extended system are $\mathcal{P}=\mathcal{Q}=\Sigma$ and satisfy

$$
\Sigma=A_{e}^{T} \Sigma A_{e}+B_{e} B_{e}^{T}, \quad \Sigma=A_{e} \Sigma A_{e}^{T}+C_{e}^{T} C_{e} .
$$

Thus the extended system is balanced in the sense of Moore [11] (see [19] for a similar result for constant dimensions). Let $T$ be the following (orthogonal) permutation matrix

$$
T=\left[\begin{array}{cccc|cccc}
I_{r_{0}} & 0 & \cdots & 0 & 0 & 0 & \cdots & 0 \\
0 & 0 & \cdots & 0 & I_{n_{0}-r_{0}} & 0 & \cdots & 0 \\
0 & I_{r_{1}} & \cdots & 0 & 0 & 0 & \cdots & 0 \\
0 & 0 & \cdots & 0 & 0 & I_{n_{1}-r_{1}} & \cdots & 0 \\
\vdots & \vdots & & \vdots & \vdots & \vdots & & \vdots \\
0 & 0 & \cdots & I_{r_{K-1}} & 0 & 0 & \cdots & 0 \\
0 & 0 & \cdots & 0 & 0 & 0 & \cdots & I_{n_{K-1}-r_{K-1}}
\end{array}\right]
$$

It follows that the transformed extended system

$$
\left(\widetilde{A}_{e}, \widetilde{B}_{e}, \widetilde{C}_{e}\right):=\left(T^{T} A_{e} T, T^{T} B_{e}, C_{e} T\right)
$$

is also balanced with the Gramians

$$
\widetilde{P}_{e}=\widetilde{Q}_{e}=\widetilde{\Sigma}=\left[\begin{array}{cc}
\widetilde{\Sigma}_{1} & 0 \\
0 & \widetilde{\Sigma}_{2}
\end{array}\right],
$$


where $\widetilde{\Sigma}_{1}=\operatorname{diag}\left(\Sigma_{0,1}, \ldots, \Sigma_{K-1,1}\right)$ and $\widetilde{\Sigma}_{2}=$ $\operatorname{diag}\left(\Sigma_{0,2}, \ldots, \Sigma_{K-1,2}\right)$ result according to the partition (10) of the periodic Gramians. It is known that the extended system is asymptotically stable and minimal provided the corresponding periodic system is asymptotically stable and minimal [2]. Corresponding to the above partition of Gramians we can partition the matrices of the transformed extended system as

$$
\begin{gathered}
\widetilde{A}_{e}=\left[\begin{array}{cc}
\widetilde{A}_{e, 11} & \widetilde{A}_{e, 12} \\
\widetilde{A}_{e, 21} & \widetilde{A}_{e, 22}
\end{array}\right], \quad \widetilde{B}_{e}=\left[\begin{array}{c}
\widetilde{B}_{e, 1} \\
\widetilde{B}_{e, 2}
\end{array}\right], \\
\widetilde{C}_{e}=\left[\begin{array}{ll}
\widetilde{C}_{e, 1} & \widetilde{C}_{e, 2}
\end{array}\right]
\end{gathered}
$$

and apply the results of [13] to the reduced system defined by $\left(\widetilde{A}_{e, 11}, \widetilde{B}_{e, 1}, \widetilde{C}_{e, 1}\right)$. It follows that this system is stable and minimal (reachable and observable). However this system is nothing else but $\left(Z_{\mathbf{r}} \mathcal{A}_{r}, Z_{\mathbf{r}} \mathcal{B}_{r}, \mathcal{C}_{r}\right)$, thus the reduced periodic system $\left(\mathcal{A}_{r}, \mathcal{B}_{r}, \mathcal{C}_{r},\right)$ is stable and minimal as well.

Consider now the periodic error system $\left(\mathcal{A}_{\text {err }}, \mathcal{B}_{\text {err }}, \mathcal{C}_{\text {err }},\right)$ defined by the system matrices

$$
\begin{gathered}
A_{e r r, k}=\left[\begin{array}{cc}
A_{k} & 0 \\
0 & A_{k, 11}
\end{array}\right], \quad B_{e r r, k}=\left[\begin{array}{c}
B_{k} \\
B_{k, 1}
\end{array}\right], \\
C_{e r r, k}=\left[\begin{array}{ll}
C_{k} & -C_{k, 1}
\end{array}\right]
\end{gathered}
$$

The following theorem is a generalization of the main result of [1] on guaranteed error bound.

Theorem 2 The $H_{\infty}$-norm of the periodic error system $\left(\mathcal{A}_{\text {err }}, \mathcal{B}_{\text {err }}, \mathcal{C}_{\text {err }},\right)$ defined by $(12)$ is bounded by

$$
2 \sum_{k=0}^{K-1} \operatorname{tr} \Sigma_{k, 2} .
$$

Proof. The $H_{\infty}$-norm of the stable periodic system $\left(\mathcal{A}_{e r r}, \mathcal{B}_{\text {err }}, \mathcal{C}_{e r r},\right)$ is given by the $H_{\infty}$-norm of the transfer-function matrix $G_{\text {err }}(z)$ of the corresponding extended system [2]. By straightforward permutation of state-variables of this system, it can be shown that

$$
G_{\text {err }}(z)=G(z)-G_{r}(z),
$$

where $G(z)$ and $G_{r}(z)$ are the transfer-function matrices corresponding to the extended systems defined in Theorem 1, $\left(\widetilde{A}_{e}, \widetilde{B}_{e}, \widetilde{C}_{e}\right)$ and $\left(\widetilde{A}_{e, 11}, \widetilde{B}_{e, 1}, \widetilde{C}_{e, 1}\right)$, respectively. By applying the result of [1, Theorem 2], we obtain that

$$
\left\|G(z)-G_{r}(z)\right\|_{\infty} \leq 2 \operatorname{tr} \widetilde{\Sigma}_{2}
$$

and this proves the theorem.

A finer bound based on the sum of distinct Hankelsingular values in $\Sigma_{k, 2}$ can be also derived using the results of [8, Corollary 2.7]. This bound is analogous to that of [9] for constant dimension periodic systems.

For an original minimal periodic system the reduced order model resulting by the BT method can be computed by performing first a balancing transformation and then simply truncating the trailing states such that for a given acceptable approximation error the above bound is satisfied. This method is however not applicable if the original system is non-minimal. Moreover, unnecessary accuracy loss can occur if balancing is performed for badly scaled original systems. In what follows, we discuss numerically reliable approaches to compute the matrices of the reduced model by means of appropriate truncation formulas.

\section{Square-root BT method}

For an asymptotically stable non-minimal periodic system $(\mathcal{A}, \mathcal{B}, \mathcal{C})$, the balancing transformation is not defined because the system is not completely reachable and/or not completely observable, and thus, from Proposition 1 or $2 \Sigma$ in (9) is not invertible. For such a system, a reduced order model can be computed by the BT approach by first determining a minimal realization $(\widehat{\mathcal{A}}, \widehat{\mathcal{B}}, \widehat{\mathcal{C}})$ of the original system [18] and then determining the reduced system $\left(\mathcal{A}_{r}, \mathcal{B}_{r}, \mathcal{C}_{r},\right)$ using the approach of the previous section.

We show now how it is possible to determine directly the matrices of the reduced system $\left(\mathcal{A}_{r}, \mathcal{B}_{r}, \mathcal{C}_{r},\right)$ using appropriate truncation formulas which generalize those in the standard case [15]. Let us write the singular value decomposition (9) at each time instant $k$ in the partitioned form

$$
R_{k} S_{k}^{T}=\left[\begin{array}{ll}
U_{k, 1} & U_{k, 2}
\end{array}\right]\left[\begin{array}{cc}
\Sigma_{k, 1} & 0 \\
0 & \Sigma_{k, 2}
\end{array}\right]\left[V_{k, 1} V_{k, 2}\right]^{T},
$$

where $\Sigma_{k, 1} \in \mathrm{R}^{r_{k} \times r_{k}}, U_{k, 1} \in \mathrm{R}^{n_{k} \times r_{k}}, V_{k, 1} \in \mathrm{R}^{n_{k} \times r_{k}}$ and $\Sigma_{k, 1}>0$. From the above decomposition define, with $\widetilde{\Sigma}_{1}=\operatorname{diag}\left(\Sigma_{0,1}, \ldots, \Sigma_{K-1,1}\right)$, the truncation matrices

$$
\mathcal{L}=\widetilde{\Sigma}_{1}^{-\frac{1}{2}} \mathcal{U}_{1}^{T} \mathcal{R}, \quad \mathcal{T}=\mathcal{S}^{T} \mathcal{V}_{1} \widetilde{\Sigma}_{1}^{-\frac{1}{2}}
$$

Then the matrices of the reduced system can be computed using the truncation formulas

$$
\mathcal{A}_{r}=\sigma \mathcal{L} \mathcal{A} \mathcal{T}, \quad \mathcal{B}_{r}=\sigma \mathcal{L B}, \quad \mathcal{C}_{r}=\mathcal{C} \mathcal{T} .
$$

The computation of the reduced model relies exclusively on square-root information (the Cholesky factors of Gramians) and this leads to a guaranteed enhancement of the overall numerical accuracy of computations. Note that the key computation in determining the truncation matrices $\mathcal{L}$ and $\mathcal{T}$ is the solution of the two PDPLEs (6) and (7) with time-varying dimensions directly for the Cholesky factors of the Gramians. 


\section{Balancing-free square-root BT method}

Ill-conditioned (i.e., nearly rank deficient) truncation matrices $\mathcal{L}$ and $\mathcal{T}$ can result if the original system is poorly scaled. Since $\mathcal{L} \mathcal{T}=I$, the pair $(\mathcal{L}, \mathcal{T})$ defines a projector $\mathcal{T} \mathcal{L}$, in analogy to the case of standard systems. Thus, for any invertible $\mathcal{W}$ compatible with the pair $(\mathcal{L}, \mathcal{T})$, the transformed pair of truncation matrices

$$
(\widetilde{\mathcal{L}}, \widetilde{\mathcal{T}})=\left(\mathcal{W}^{-1} \mathcal{L}, \mathcal{T} \mathcal{W}\right)
$$

defines the same projected system but in a different coordinate form. To avoid potential accuracy losses, an alternative to balancing is to use a balancing-free approach to determine the truncation matrices. In standard case, such a method has been proposed in [14], involving the computation of appropriate left/right invariant subspaces of the product $\mathcal{P} \mathcal{Q}$. A square-root balancing-free approach for the periodic case can be devised analogously as in case of standard systems [16], by combining the square-root method with a balancing-free method. Consider the QR-decompositions

$$
\mathcal{S}^{T} \mathcal{V}_{1}=\widetilde{\mathcal{T}} \mathcal{X}, \quad \mathcal{R}^{T} \mathcal{U}_{1}=\widetilde{\mathcal{Z}} \mathcal{Y}
$$

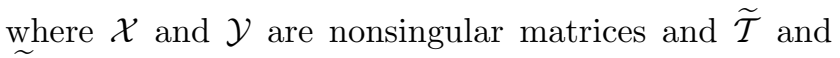
$\widetilde{\mathcal{Z}}$ are matrices with orthonormal columns. With the already computed $\widetilde{\mathcal{T}}$ we define the corresponding $\widetilde{\mathcal{L}}$ as

$$
\widetilde{\mathcal{L}}=\left(\widetilde{\mathcal{Z}}^{T} \widetilde{\mathcal{T}}\right)^{-1} \widetilde{\mathcal{Z}}^{T}
$$

We have the following result.

\section{Theorem 3 The periodic system}

$$
\left(\widetilde{\mathcal{A}}_{r}, \widetilde{\mathcal{B}}_{r}, \widetilde{\mathcal{C}}_{r}\right):=(\sigma \widetilde{\mathcal{L}} \mathcal{A} \widetilde{\mathcal{T}}, \sigma \widetilde{\mathcal{L}} \mathcal{B}, \mathcal{C} \widetilde{\mathcal{T}})
$$

with $\widetilde{\mathcal{L}}$ and $\widetilde{\mathcal{T}}$ defined in (17) and (18) is Lyapunovsimilar to the reduced system $\left(\mathcal{A}_{r}, \mathcal{B}_{r}, \mathcal{C}_{r}\right)$ obtained by the square-root approach.

Proof. We observe that using (17) and (18), we can relate the truncation matrices in (14) with $\widetilde{\mathcal{L}}$ and $\widetilde{\mathcal{T}}$ as

$$
\mathcal{L}=\widetilde{\Sigma}^{-\frac{1}{2}} \mathcal{Y}^{T} \widetilde{\mathcal{Z}}^{T}=\widetilde{\Sigma}^{-\frac{1}{2}} \mathcal{Y}^{T}\left(\widetilde{\mathcal{Z}}^{T} \widetilde{\mathcal{T}}\right) \widetilde{\mathcal{L}}, \quad \mathcal{T}=\widetilde{\mathcal{T}} \mathcal{X} \widetilde{\Sigma}^{-\frac{1}{2}} .
$$

Thus, according to $(16)\left(\widetilde{\mathcal{A}}_{r}, \widetilde{\mathcal{B}}_{r}, \widetilde{\mathcal{C}}_{r}\right)$ and $\left(\mathcal{A}_{r}, \mathcal{B}_{r}, \mathcal{C}_{r}\right)$ are Lyapunov-similar provided

$$
\mathcal{W}=\widetilde{\Sigma}^{-\frac{1}{2}} \mathcal{Y}^{T}\left(\widetilde{\mathcal{Z}}^{T} \tilde{\mathcal{T}}\right)
$$

is the corresponding similarity transformation having as its inverse $\mathcal{X} \widetilde{\Sigma}^{-\frac{1}{2}}$. This follows by checking that

$$
\widetilde{\Sigma}^{-\frac{1}{2}} \mathcal{Y}^{T}\left(\widetilde{\mathcal{Z}}^{T} \tilde{\mathcal{T}}\right) \mathcal{X} \widetilde{\Sigma}^{-\frac{1}{2}}=\widetilde{\Sigma}^{-\frac{1}{2}} \mathcal{U}_{1}^{T} \mathcal{R S}^{T} \mathcal{V}_{1} \widetilde{\Sigma}^{-\frac{1}{2}}=I
$$

Since the balancing-free square-root approach always constructs well-conditioned truncation matrices, the use of this method for poorly scaled systems leads usually to an improvement of the overall computational accuracy.

\section{Numerical issues}

The main computational problem to perform model reduction of periodic systems by using the BT approach consists in solving a pair of PDPLEs of the forms (6) and (7) directly for the Cholesky factors of the Gramians. Numerical algorithms for this computations have been proposed in [17] for the case of constant dimensions, and in [18] for the case of time-varying dimensions. Both algorithms represent extensions of the method for standard systems proposed by Hammarling [7]. The new methods rely on an initial reduction of the periodic Lyapunov equations to simpler forms by using the periodic Schur decomposition (PSD) of the product of square matrices $\Phi_{A}(K, 0)$ [3] or the extended PSD of a square product $\Phi_{A}(K, 0)$ of possibly rectangular matrices [18]. The reduced equations are then solved by using special forward substitution algorithms. Note that to compute the Cholesky factors of the reachability and observability Gramians a single computation of the (extended) PSD of the monodromy matrix $\Phi_{A}(K, 0)$ is sufficient. In this way, the cost to solve the two PDPLEs (6) and (7) is almost the same as the cost of solving a single PDPLE. Important computational subproblems are in this context the efficient and numerically stable solution of order one or order two PDPLEs and of low order discrete periodic Sylvester equations. Computational approaches for these subproblems are described in detail in [17]. An alternative to the Schur approach to solve periodic Lyapunov equations is to extend the iterative square-root technique proposed for constant dimensions in [17, Algorithm 5] to the time-varying case.

\section{Conclusions}

In this paper we discussed the order reduction of periodic systems using the BT approach. From theoretical point of view we provide results which generalize analogue ones for standard discrete-time systems for the stability and minimality of the reduced system as well as for the guaranteed approximation error bound. The computation of the reduced system matrices can be done by using numerically sound approaches: the square-root method or the balancing-free square-root methods. Both methods use exclusively square-root information in form of Cholesky factors of the Gramians and therefore have guaranteed enhanced computational accuracy. We believe that these methods represent completely satisfactory numerical approaches to solve periodic BT model reduction problems and can serve as basis for robust numerical implementations. The computational complexity of our approach to the periodic model reduction problem is $O\left(K \bar{n}^{3}\right)$, where $\bar{n}=\max \left\{n_{i}\right\}$. Note that the computational complexity of an LMIs-based approach, although still polynomial 
in $\bar{n}$, is at least with one order of magnitude higher.

The proposed setting with time-varying dimensions for the BT model reduction of periodic systems seems to be the only proper way to address the periodic model reduction problem. Note that time-varying orders of the reduced system matrices result always when, in analogy to the standard systems case, the separation of Hankel singular values is determined by a given error tolerance. This contrasts to [20], where the interlacing property of the periodic Hankel singular values has been assumed. Note also that methods based on balanced forms are restricted to periodic systems which possess balanced minimal realizations with constant dimensions. It seems that ignoring the time-varying dimension aspect was the explanation for the lack of a complete theory of model reduction for periodic systems.

Remark. During the preparation of the final manuscript, we were aware of the recent work [10], which partly parallels our results (Theorems 1 and 2). However, in contrast to our approach, the method of [10] does not address the reduction of non-minimal periodic systems.

\section{References}

[1] U. M. Al-Saggaf and G. F. Franklin. An error bound for a discrete reduced order model of a linear multivariable system. IEEE Trans. Autom. Control, 32:815-819, 1987.

[2] S. Bittanti and P. Colaneri. Analysis of discretetime linear periodic systems. In C. T. Leondes, editor, Digital Control and Signal Processing Systems and Techniques, volume 78 of Control and Dynamics Systems, pages 313-339. Academic Press, 1996.

[3] A. W. Bojanczyk, G. Golub, and P. Van Dooren. The periodic Schur decomposition. Algorithms and applications. In F. T. Luk, editor, Proceedings SPIE Conference, volume 1770, pp. 31-42, July 1992.

[4] P. Colaneri and S. Longhi. The realization problem for linear periodic systems. Automatica, 31:775$779,1995$.

[5] I. Gohberg, M. A. Kaashoek, and L. Lerer. Minimality and realization of discrete time-varying systems. Operator Theory: Advances and Applications, 56:261296,1992 .

[6] O. M. Grasselli and S. Longhi. Pole-placement for nonreachable periodic discrete-time systems. Math. Control Signals Syst., 4:439-455, 1991.

[7] S. J. Hammarling. Numerical solution of the stable, non-negative definite Lyapunov equation. IMA J. Numer. Anal., 2:303-323, 1982.

[8] D. Hinrichsen and A. J. Pritchard. An improved error estimate for reduced-order models of discrete-time systems. IEEE Trans. Autom. Control, 35:317-320, 1990.

[9] S. Lall, C. Beck, and G. Dullerud. Guaranteed error bounds for model reduction of linear time-varying systems. In Proc. ACC'98, 1998.

[10] S. Longhi, and G. Orlando. Balanced reduction of linear periodic systems. Kybernetika, 35:737-751, 1999.

[11] B. C. Moore. Principal component analysis in linear system: controllability, observability and model reduction. IEEE Trans. Autom. Control, AC-26:17-32, 1981.

[12] B. Park and E. I. Verriest. Canonical forms for discrete-time periodically time varying systems and a control application. In Proc. of CDC'89, Tampa, pp. 1220-1225, 1989.

[13] L. Pernebo and L. M. Silverman. Model reduction via balanced state space representations. IEEE Trans. Autom. Control, 27:382-387, 1982.

[14] M. G. Safonov and R. Y. Chiang. A Schur method for balanced-truncation model reduction. IEEE Trans. Autom. Control, 34:729-733, 1989.

[15] M. S. Tombs and I. Postlethwaite. Truncated balanced realization of a stable non-minimal state-space system. Int. J. Control, 46:1319-1330, 1987.

[16] A. Varga. Efficient minimal realization procedure based on balancing. In A. El Moudni, P. Borne, and S. G. Tzafestas, editors, Prepr. of IMACS Symp. on Modelling and Control of Technological Systems, vol. 2, pp. 42-47, 1991.

[17] A. Varga. Periodic Lyapunov equations: some applications and new algorithms. Int. J. Control, 67:6987, 1997.

[18] A. Varga. Balancing related methods for minimal realization of periodic systems. Systems \& Control Lett., 36:339-349, 1999.

[19] E. I. Verriest and U. Helmke. Periodic balanced realizations. In Proc. of IFAC System Structure and Control Symp., Nantes, France, 1998.

[20] B. Xie, R. K. A. V. Aripirala, and V. Syrmos. Model reduction of linear discrete-time periodic systems using hankel-norm approximations. In Proc. 13th IFAC Congress, San Francisco, USA, pp. 245-250, 1996. 